\section{Combination Therapy of Abatacept and Anakinra in Children with Refractory Systemic Juvenile Idiopathic Arthritis: A Retrospective Case Series}

To the Editor:

Novel biological agents have provided improved treatment options for children with systemic juvenile idiopathic arthritis (sJIA). Anakinra, an interleukin 1 receptor antagonist, has been shown to effectively treat sJIA $^{1,2,3,4,5}$; however, some patients may require multiple painful daily injections to control their disease. Another biologic, abatacept, selectively modulates the CD80/CD86:CD28 costimulatory signal necessary for T cell activation $^{6}$ and has been shown to be effective in treating patients with sJIA who have a polyarticular course ${ }^{7}$. Rheumatologists are generally hesitant to combine biologics because of studies involving adults with rheumatoid arthritis showing no improvement when combining anticytokine biologics and an increased risk of infection ${ }^{8,9}$. However, Weinblatt, et al actually reported a lower incidence of severe adverse events in a small group of patients receiving anakinra and abatacept than in patients receiving anakinra and placebo, although the efficacy of this combination has not been formally evaluated ${ }^{8}$.

Based on the available literature regarding the effectiveness of anakinra and abatacept in the treatment of sJIA independently, we have begun using these agents concurrently in selected patients with refractory disease. The mechanism of action of abatacept (costimulatory blockade) was deemed notably different from cytokine inhibition, and we postulated that combination therapy would have an additive or synergistic benefit for these steroid-dependent patients with refractory sJIA because of its unique mechanism of action. We performed a retrospective review of children with difficult-to-treat sJIA treated simultaneously with anakinra and abatacept to report the efficacy and safety of this combination.

All patients at the Children's Hospital of Alabama, Birmingham, AL, USA, diagnosed with sJIA by a treating board-certified pediatric rheumatologist and receiving combination therapy with abatacept and anakinra were eligible for this study. After receiving institutional review board approval, 4 patients were identified and their records were reviewed. Data were collected on a standard form regarding demographics, laboratory values, joint count, and any infectious or other adverse effects. All 4 patients are female, and 2 are siblings. Three are African American and 1 is of European descent.
Patient 1 is a 6-year-old African American female who developed symptoms of sJIA at the age of 8 months. She eventually received anakinra, methotrexate (MTX), and high-dose corticosteroids but continued to have breakthrough fever, arthritis, rash, and low-grade macrophage activation syndrome (MAS). Abatacept was added to that treatment regimen 4 years after initial symptoms for treatment of arthritis. Her fevers improved from 2-3 times per week to only occurring the week prior to abatacept infusion. Her initial abatacept dose was increased to $18 \mathrm{mg} / \mathrm{kg}$ every 3 weeks, for greater arthritis control (Table 1). Her erythrocyte sedimentation rate (ESR) had normalized to $18 \mathrm{~mm} / \mathrm{h}$ at last followup after being 60-100 $\mathrm{mm} / \mathrm{h}$ throughout the previous year.

Patient 2 is a 12-year-old white female who developed sJIA at 10 years of age. She initially responded well to corticosteroids and anakinra, but then developed chronic low-grade MAS with concurrent cutaneous vasculitis. She was treated aggressively with pulse methylprednisolone, intravenous immunoglobulin (IVIG), cyclophosphamide, cyclosporine, and 400 mg per day of anakinra. She developed marked corticosteroid adverse effects including steroid myopathy, diabetes mellitus, significant hypertension, poor wound healing, and marked weight gain. She eventually improved and tolerated an initial steroid taper; abatacept was added to this treatment regimen as a less toxic steroid-sparing agent 2 years after initial symptoms. Cyclosporine, IV cyclophosphamide, and IVIG were all discontinued about 1 month prior to starting abatacept. She successfully discontinued prednisone 2 months after starting abatacept and was able to reduce her anakinra dosage to $100 \mathrm{mg}$ per day. Her disease remains controlled on anakinra and abatacept with no further hospitalizations for MAS.

Patient 3 is an 8-year-old African American female who developed sJIA at the age of 2 years. She responded favorably to corticosteroids, MTX, and anakinra, although her ESR remained persistently elevated, ranging from 51 to $120 \mathrm{~mm} / \mathrm{h}$ since her diagnosis. Six years after initial symptoms, abatacept was added to her treatment regimen in an effort to control her destructive arthritis, which has subsequently improved from 25 affected joints prior to starting abatacept to only 8 joints at last followup. Her ESR began to normalize 2 months after her initial abatacept infusion, having been persistently elevated for the entire 6 years since her diagnosis; it was $19 \mathrm{~mm} / \mathrm{h}$ at last followup.

Patient 4 is a 6-year-old African American female (younger sibling of Patient 3) who developed sJIA at the age of 2 years. She responded favorably to a combination of corticosteroids, MTX, and anakinra. Abatacept

Table 1. Clinical summary of patients with sJIA treated with abatacept and anakinra.



\footnotetext{
* Months since onset of initial sJIA symptoms to start of abatacept. ${ }^{\dagger}$ Corticosteroid dosing was converted into prednisone equivalent and calculated as $\mathrm{mg} / \mathrm{kg} /$ day. Ank: anakinra; CS: corticosteroids; MTX: methotrexate (subcutaneous); Abt: abatacept; IVIG: intravenous immunoglobulin; MAS: macrophage activation syndrome; ESR: erythrocyte sedimentation rate; sJIA: systemic juvenile idiopathic arthritis; CYC: cyclophosphamide.
} 
was added to that treatment regimen 3 years after initial symptoms in response to persistent arthritis and inflammation. Her arthritis went from 6 affected joints to none at last followup, and her ESR, which averaged 60 $\mathrm{mm} / \mathrm{h}$ prior to abatacept, began to steadily decrease 3 months after abatacept initiation and was $29 \mathrm{~mm} / \mathrm{h}$ at last followup, the lowest it has been since diagnosis.

To our knowledge, ours is the first description of the concurrent use of anakinra and abatacept in children with sJIA. This combination therapy appears to be beneficial in these 4 steroid-dependent patients with refractory sJIA, allowing dose reduction of both anakinra and corticosteroids while improving arthritis joint count, as well as controlling systemic features of disease (Table 1). With 8-17 months of followup since initiation of abatacept, no infusion reactions, significant infections, or other notable adverse effects have been observed in any of the patients. Although the evidence is anecdotal at present, a controlled clinical trial to assess the efficacy and safety of this combination therapy in children with refractory sJIA is warranted.

JESSICA L. RECORD, BS; TIMOTHY BEUKELMAN, MD, MSCE, Assistant Professor; RANDY Q. CRON, MD, PhD, Professor, Children's Hospital of Alabama, University of Alabama at Birmingham, Department of Pediatrics, Division of Pediatric Rheumatology. Address correspondence to Dr. R.Q. Cron, Children's Hospital of Alabama/University of Alabama at Birmingham, Children's Park Place, Ste. 210,

1601 4th Avenue South, Birmingham, AL 35233, USA.

E-mail: rcron@peds.uab.edu

\section{REFERENCES}

1. Pascual V, Allantaz F, Arce E, Punaro M, Banchereau J. Role of interleukin-1 (IL-1) in the pathogenesis of systemic onset juvenile idiopathic arthritis and clinical response to IL-1 blockade. J Exp Med 2005;201:1479-86.
2. Verbsky JW, White AJ. Effective use of the recombinant interleukin 1 receptor antagonist anakinra in therapy resistant systemic onset juvenile rheumatoid arthritis. J Rheumatol 2004;31:2071-5.

3. Lequerre T, Quartier P, Rosellini D, Alaoui F, De Bandt M, Mejjad $\mathrm{O}$, et al. Interleukin-1 receptor antagonist (anakinra) treatment in patients with systemic-onset juvenile idiopathic arthritis or adult onset Still disease: preliminary experience in France. Ann Rheum Dis 2008;67:302-8.

4. Zeft A, Hollister R, LaFleur B, Sampath P, Soep J, McNally B, et al. Anakinra for systemic juvenile arthritis: the Rocky Mountain experience. J Clin Rheumatol 2009;15:161-4.

5. Ohlsson V, Baildam E, Foster H, Jandial S, Pain C, Strike H, et al. Anakinra treatment for systemic onset juvenile idiopathic arthritis (SOJIA). Rheumatology 2008;47:555-6.

6. Cron RQ. A signal achievement in the treatment of arthritis. Arthritis Rheum 2005;52:2229-32.

7. Ruperto N, Lovell DJ, Quartier P, Paz E, Rubio-Perez N, Silva CA, et al. Long-term safety and efficacy of abatacept in children with juvenile idiopathic arthritis. Arthritis Rheum 2010;62:1792-802.

8. Weinblatt M, Combe B, Covucci A, Aranda R, Becker JC, Keystone E. Safety of the selective costimulation modulator abatacept in rheumatoid arthritis patients receiving background biologic and nonbiologic disease-modifying antirheumatic drugs: A one-year randomized, placebo-controlled study. Arthritis Rheum 2006;54:2807-16.

9. Genovese MC, Cohen S, Moreland L, Lium D, Robbins S, Newmark R, et al. Combination therapy with etanercept and anakinra in the treatment of patients with rheumatoid arthritis who have been treated unsuccessfully with methotrexate. Arthritis Rheum 2004;50:1412-9.

J Rheumatol 2011;38:1; doi:10.3899/jrheum.100726 